\title{
Simulation of Hydrogen-Induced Reconstruction of Oxygen on $\operatorname{Pd}(111)$
}

\author{
V. Petrauskas and E.E. Tornau \\ Semiconductor Physics Institute \\ Goštauto 11, LT-01108, Vilnius, Lithuania
}

\begin{abstract}
The oxygen and hydrogen atoms co-adsorption experiment on $\operatorname{Pd}(111)$ is modelled using kinetic Monte Carlo method. We consider $\mathrm{O}-\mathrm{O}, \mathrm{H}-\mathrm{H}$, and $\mathrm{O}-\mathrm{H}$ interactions and several kinetic processes (diffusion, surface and subsurface $\mathrm{H}$-atoms exchange and water-formation reaction) leading to the phase transition $(2 \times 2)_{\mathrm{O}} \rightarrow(\sqrt{3} \times \sqrt{3})_{\mathrm{O}}$ with increase in H-coverage. We also demonstrate how reverse phase transition $(\sqrt{3} \times \sqrt{3})_{\mathrm{O}} \rightarrow(2 \times 2)_{\mathrm{O}}$ occurs due to hydrogen dissolution into subsurface in the absence of hydrogen gas. At higher temperature we observe the disappearance of $(2 \times 2)_{\mathrm{O}}$ oxygen islands due to water-formation reaction.
\end{abstract}

PACS numbers: 68.43.Fg, 68.43.Hn, 68.43.Mn, 64.60.Cn

\section{Introduction}

The studies of $\mathrm{O}$ - and $\mathrm{H}$-atoms co-adsorption on transition metal surfaces are stimulated by their importance for heterogeneous catalysis, corrosion and even phase transition physics. Adsorbed $\mathrm{H}$-atoms can drastically change the structure and properties of oxidized surfaces. Hydrogen adsorbed on oxidized $\operatorname{Pd}(111)$ enhances the diffusion of O-atoms and leads to structural phase transition $(2 \times 2)_{\mathrm{O}} \rightarrow(\sqrt{3} \times \sqrt{3})_{\mathrm{O}}$. This phase transition occurs [1] in temperature range (150-200 K) wherein O-atoms have very low mobility (activation energy for diffusion $0.4-0.5 \mathrm{eV}[2]$ ) when there are no $\mathrm{H}$ or $\mathrm{CO}$ atoms. Above $205 \mathrm{~K}$ oxygen structure transforms back to $(2 \times 2)_{\mathrm{O}}$, if adsorption of additional H-atoms is terminated. Only with further increase in temperature the ordered oxygen islands shrink in size evidencing water formation caused by reaction between co-adsorbed $\mathrm{O}$ and $\mathrm{H}$ [1]. Here we perform kinetic Monte Carlo simulation of these transitions in a very narrow temperature range $(200-220 \mathrm{~K})$ accounting for the $\mathrm{O}-\mathrm{O}, \mathrm{H}-\mathrm{H}$, and $\mathrm{O}-\mathrm{H}$ interactions and rates of main dynamic processes.

\section{Kinetic processes and results}

For our simulations we use the pair algorithm approach [3], since the model contains bimolecular steps, e.g., diffusion and reaction. The main idea of the algo- 
rithm is to consider two nearest neighbor (NN) sites simultaneously and account for all possible processes that might occur on this pair of sites. The processes considered in our simulation on a hexagonal lattice of $48 \times 48 \mathrm{Pd}$ atoms are $\mathrm{O}$ and $\mathrm{H}$ diffusion via $\mathrm{NN}$ fcc adsorption sites, $\mathrm{H}$ dissolution to subsurface and segregation back to the surface, as well as the reaction between $\mathrm{O}$ and $\mathrm{H}$ in the NN sites.

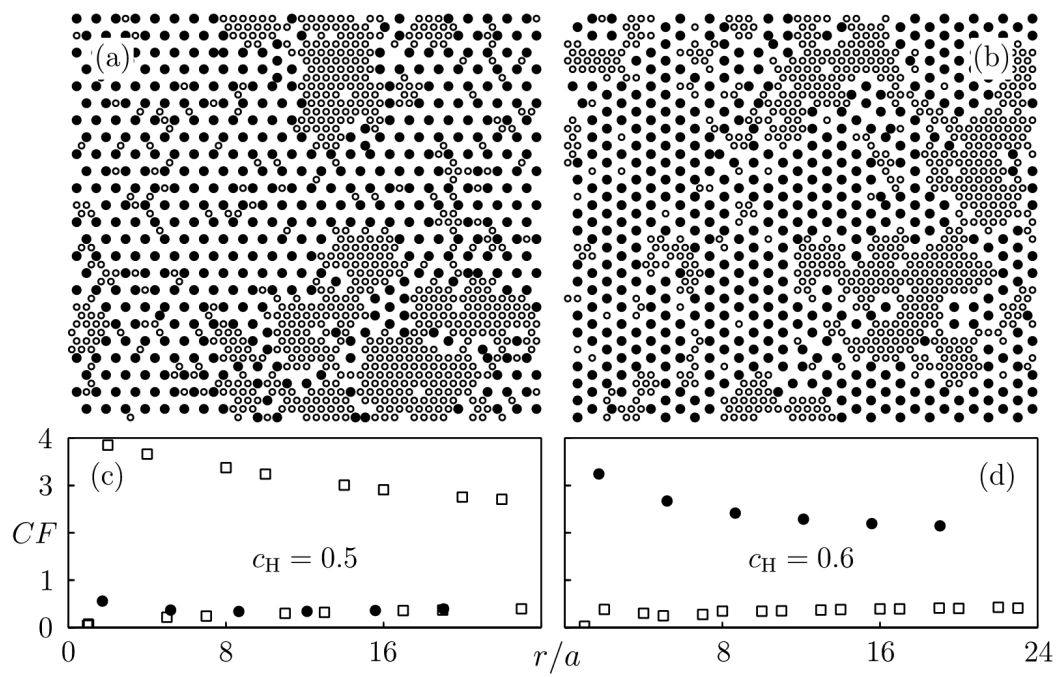

Fig. 1. Snapshots of our calculations at $T=200 \mathrm{~K}$ and $c_{\mathrm{H}}=0.5$ (a) and $c_{\mathrm{H}}=0.6$ (b). O-atoms are represented by filled and H-atoms - by open circles. Oxygen correlation functions (c) and (d) at the corresponding coverages: black dots represent the CF of the $(\sqrt{3} \times \sqrt{3})_{\mathrm{O}}$ phase, open squares $-\mathrm{CF}$ of the $(2 \times 2)_{\mathrm{O}}$ phase.

Performing simulation with the initial O-coverage $c_{\mathrm{O}}=0.2$ and varying $\mathrm{H}$ coverage $c_{\mathrm{H}}$ from 0.4 to 0.7 we obtain $(2 \times 2)_{\mathrm{O}} \rightarrow(\sqrt{3} \times \sqrt{3})_{\mathrm{O}}$ phase transition (see snapshots and pair correlation functions $(\mathrm{CF})$ at $T=200 \mathrm{~K}$ in Fig. 1). CF is defined as $c_{\mathrm{OO}}(r) / c_{\mathrm{O}}^{2}$, where $c_{\mathrm{OO}}(r)$ is concentration of $\mathrm{O}-\mathrm{O}$ pairs at a distance $r$. In the mean-field approximation $c_{\mathrm{OO}}(r)=c_{\mathrm{O}}^{2}$, which leads to $\mathrm{CF}$ equal to 1. The condition $\mathrm{CF}\left(r_{\mathrm{c}}\right)=1$ allows us to estimate the oxygen domain radius $r_{\mathrm{c}}$. $\mathrm{O}$ and $\mathrm{H}$ diffusion rates are taken in the activated form with parameters $\nu_{0}^{\mathrm{O}}=10^{12} \mathrm{~s}^{-1}, E_{a}^{\mathrm{O}}=0.4 \mathrm{eV}[2]$ and $\nu_{0}^{\mathrm{H}}=10^{7} \mathrm{~s}^{-1}, E_{a}^{\mathrm{H}}=0.1 \mathrm{eV}$ respectively, and the following set of interaction parameters $v_{i}$ is chosen: repulsion of the NN atoms $\left(v_{1}^{\mathrm{O}-\mathrm{O}}=160 \mathrm{meV}, v_{1}^{\mathrm{H}-\mathrm{H}}=16 \mathrm{meV}\right.$, and $\left.v_{1}^{\mathrm{O}-\mathrm{H}}=80 \mathrm{meV}\right)$ and also the repulsion of the next- $\mathrm{NN}\left(v_{2}^{\mathrm{O}-\mathrm{O}}=16 \mathrm{meV}\right)$ and attraction of the $3 \mathrm{rd}-\mathrm{NN}$ $\left(v_{3}^{\mathrm{O}-\mathrm{O}}=-6.4 \mathrm{meV}\right.$ taken for stability of the $(2 \times 2)_{\mathrm{O}}$ structure $)$ atoms [3]. We obtained $\mathrm{H}$-coverage by distributing $\mathrm{H}$-atoms on the surface fcc sites, thus avoiding extra parameters, needed when $c_{\mathrm{H}}$ is obtained from the ratio of adsorption and desorption rates. Let us note that the rates (in activated form) of $\mathrm{H}$ dissolution to subsurface $k^{\downarrow}$ and segregation back to the surface $k^{\uparrow}$ are chosen to be more or less 

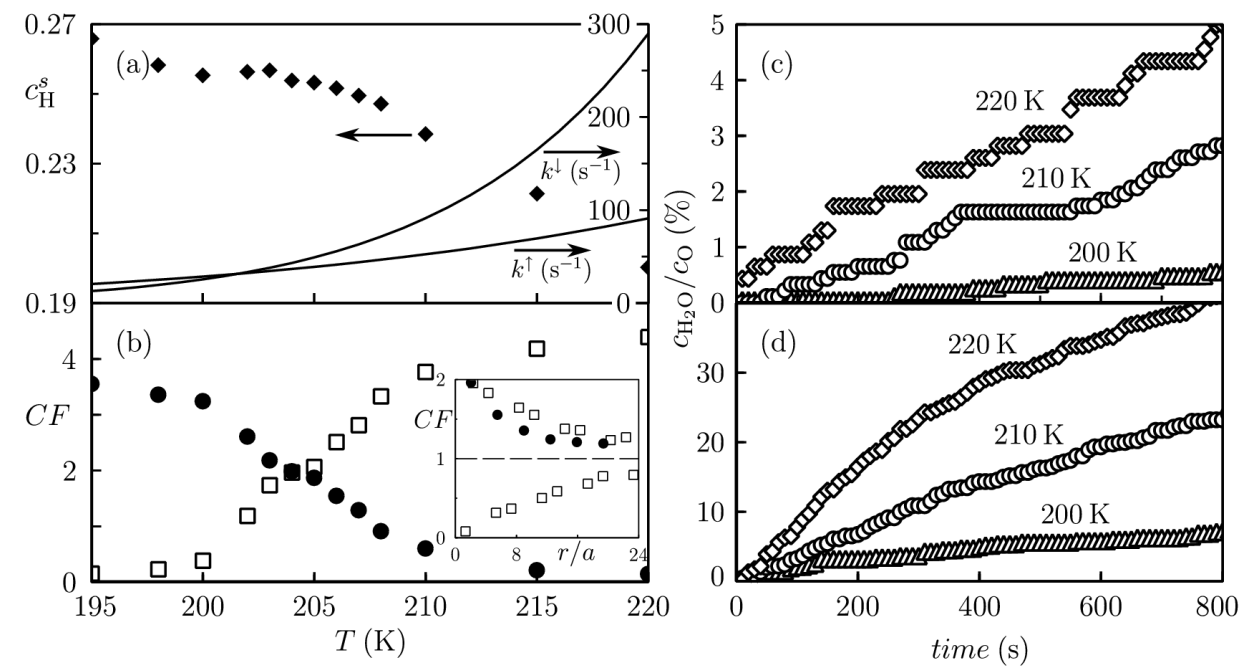

Fig. 2. Temperature dependence of surface hydrogen coverage (full diamonds) and chosen $k^{\downarrow}$ and $k^{\uparrow}$ rates (lines) (a) and CFs (b): black dots represent CF of the $(\sqrt{3} \times \sqrt{3})_{\mathrm{O}}$ phase, open squares - $\mathrm{CF}$ of the $(2 \times 2)_{\mathrm{O}}$ phase. Inset in (b): oxygen $\mathrm{CF}$ vs. distance at $T=205 \mathrm{~K}$. Size of oxygen domain $r_{(\sqrt{3} \times \sqrt{3})_{\mathrm{O}}} \approx r_{(2 \times 2)_{\mathrm{O}}}=L / 2$. Increase in $c_{\mathrm{H}_{2} \mathrm{O}}$ with time at different values of temperature when oxygen reacts only with surface hydrogen (c) and oxygen reacts with both surface and subsurface hydrogen (d).

equal at $T=200 \mathrm{~K}\left(k_{0}^{\downarrow}=10^{13} \mathrm{~s}^{-1}, E_{a}^{\downarrow}=0.46 \mathrm{eV}\right.$ and $k_{0}^{\uparrow}=10^{7} \mathrm{~s}^{-1}, E_{a}^{\uparrow}=0.22$ $\mathrm{eV}$ ) giving $\mathrm{H}$-coverages of subsurface and surface 0.34 and 0.26 , respectively, at total $c_{\mathrm{H}}=0.6$. H-atoms cannot diffuse in the subsurface, but they can segregate back to the surface with probability proportional to rate of segregation.

When the phase $(\sqrt{3} \times \sqrt{3})_{\mathrm{O}}$ is achieved, the uptake of hydrogen is terminated in experiment [1]. Assuming the system with $c_{\mathrm{H}}=0.6$ (as in Fig. 1b) and increasing temperature, we have found a relative increase in $k^{\downarrow}$ (see Fig. 2a). At $205 \mathrm{~K}$ when $k^{\downarrow} / k^{\uparrow}=1.25$, it causes the reverse transition $(\sqrt{3} \times \sqrt{3})_{\mathrm{O}} \rightarrow(2 \times 2)_{\mathrm{O}}$ due to a slight $\mathrm{H}$ dissolution to subsurface (see $T$-dependence of surface $c_{\mathrm{H}}$ and behavior of corresponding CF in Fig. 2).

This transition is not affected by the water-formation reaction which starts to be important only above $220 \mathrm{~K}$ [1]. Therefore, we had to choose the reaction rate $k^{\mathrm{r}}=k_{0}^{\mathrm{r}} \exp \left(-E_{a}^{\mathrm{r}} / k_{\mathrm{B}} T\right)$ which would be negligible at $200 \mathrm{~K}$ and at the same time it would not destroy the $(\sqrt{3} \times \sqrt{3})_{\mathrm{O}}$ structure. Assuming in the beginning that the reaction proceeds only between surface $\mathrm{O}$ and $\mathrm{H}$ in the NN sites, we temporarily excluded dissolution and segregation processes and determined such reaction parameters: $E_{a}^{\mathrm{r}}=0.35 \mathrm{eV}$ and $k_{0}^{\mathrm{r}}=10^{5} \mathrm{~s}^{-1}$. This is the rate given for the first reaction step $(\mathrm{O}+\mathrm{H} \rightarrow \mathrm{OH})$ which is known [4] to be rate limiting. We also assumed the infinite rate for the second step of reaction. To compare, the parameter values $E_{a}^{\mathrm{r}}=0.3 \mathrm{eV}$ and $k_{0}^{\mathrm{r}}=8.6 \times 10^{6} \mathrm{~s}^{-1}$ were estimated from 
experiment [1]. In Figs. 2c and d we show the time dependence of ratio of reacted oxygen atoms to initial number of oxygen atoms at different values of temperature when all processes are considered. At $T=200 \mathrm{~K}$ the reaction does not play important role in phase formation, but it becomes more important at $210 \mathrm{~K}$ and higher temperature. Accounting also for the reaction (with the same $k^{\mathrm{r}}$ ) between subsurface $\mathrm{H}$ and surface $\mathrm{O}$ sitting just above it leads to drastic increase in $c_{\mathrm{H}_{2} \mathrm{O}}$ up to $5-10$ times.

In conclusion, it is shown by kinetic Monte Carlo calculation that the phase transition $(2 \times 2)_{\mathrm{O}} \rightarrow(\sqrt{3} \times \sqrt{3})_{\mathrm{O}}$ can be obtained with increase in H-coverage when the interactions between $\mathrm{O}-\mathrm{O}, \mathrm{O}-\mathrm{H}$, and $\mathrm{H}-\mathrm{H}$ are repulsive, and the rates of dynamic processes are carefully chosen. In this case it is also possible to obtain the reverse phase transition due to hydrogen dissolution to subsurface. We also observe disappearance of oxygen islands due to water-formation reaction. Both these processes are experimentally found in the limits of a very narrow temperature range $(200-220 \mathrm{~K})$.

\section{Acknowledgments}

This work was partly supported by project MULTIMA (No. C-18/2007) of Lithuanian State Science and Studies Foundation.

\section{References}

[1] T. Mitsui, M.K. Rose, E. Fomin, D.F. Ogletree, M. Salmeron, Surf. Sci. 511, 259 (2002); J. Chem. Phys. 117, 5855 (2002).

[2] M.K. Rose, A. Borg, J.C. Dunphy, T. Mitsui, D.F. Ogletree, M. Salmeron, Surf. Sci. 561, 69 (2004).

[3] E.E. Tornau, V. Petrauskas, G. Zvejnieks, Catalysis Today 116, 62 (2006).

[4] T. Engle, H. Kuipers, Surf. Sci. 90, 181 (1979). 